

\section{Propuesta para la implementación de un currículo de la Geografía en Colombia}

Proposta para a implementação de uma estrutura curricular da Geografia na Colômbia

Proposal for the Implementation of a Geography Curriculum in Colombia

Mario Fernando Hurtado Beltrán*

Olga Lucía Romero Castro**

Luis Guillermo Torres Pérez***

\begin{abstract}
Resumen
En Colombia la educación geográfica, enmarcada en la política educativa, ha tenido cambios significativos. Se ha dado la transición de área autónoma a un área integrada con la historia, la democracia y la Constitución política, bajo la denominación de Ciencias Sociales, a partir de 1994 con la Ley 115 de Educación y, recientemente, con la expedición de la Ley 1874 del 2017, que le dio a la historia un estatus diferente al de las demás ciencias sociales escolares. Estos cambios evidencian una geografía escolar disminuida y cada vez más difuminada. Desde el Grupo Interinstitucional de Investigación Geopaideia, buscando responder al porqué y al para qué de la educación geográfica escolar, se han sistematizado algunas experiencias cuyo propósito ha sido definir unas temáticas curriculares para la enseñanza de la geografía. El presente artículo es producto de estas reflexiones y de la práctica de los tres autores en políticas educativas, currículo, formación docente y enseñanza escolar.
\end{abstract}

\section{Palabras clave}

Políticas educativas en geografía, currículo escolar, formación de licenciados en Ciencias Sociales, materiales de texto, contenidos geográficos.

\footnotetext{
* Universidad Pedagógica Nacional.

** Universidad del Tolima.

*** Universidad Pedagógica Nacional.
} 
Propuesta para la implementación de un currículo de la Geografía en Colombia Mario Fernando Hurtado Beltrán / Olga Lucía Romero Castro / Luis Guillermo Torres Pérez /

\section{Resumo}

Na Colômbia, a educação geográfica, enquadrada na política educacional, sofreu mudanças significativas, mas com pouco apoio curricular e formativo. A transição da área autônoma para uma área integrada com a história, a democracia e a constituição política sob o nome de ciências sociais, a partir de 1994 com a Lei 115 da Educação e, recentemente, a Lei 1874 de 2017, da qual é dada para a história um status diferente das outras ciências sociais da escola, elas mostram uma geografia escolar reduzida e cada vez mais turvas. Do Grupo de Pesquisa Interinstitucional Geopaideia, procurando responder o porquê? e para quê? Na educação escolar geográfica, algumas experiências foram sistematizadas, cujo propósito foi definir disciplinas curriculares para o ensino da geografia. Este artigo é o produto dessas reflexões e da prática dos três autores em políticas educacionais, currículo, formação de professores e educação escolar.

\section{Abstract}

In Colombia, geographic education, in the framework of an educational policy, has experienced significant changes, with little curricular and formative support. It has undergone a transition from an autonomous to an integrated area-along with history, democracy and the political Constitution-under the name of social sciences, starting in 1994 with Law 115 of Education, and recently, with Law 1874 of 2017-which assigns History a different status to the other school social sciences-. Such changes show a diminished and increasingly blurred school geography. The Inter-Institutional Research Group Geopaideia, seeking to answer the why and what in school geographical education, has systematized some experiences aimed at defining curricular topics for the teaching of geography. This article is the product of these reflections and the practice of the three authors in educational policies, curriculum, teacher training, and school education.

\section{Palavras chaves}

Políticas educacionais em geografia, currículo escolar, formação de graduados em Ciências Sociais, materiais de texto, conteúdos geográficos.

\section{Keywords}

Educational policies in geography, school curriculum, Social Science teachers' training, text materials, geographical contents. 


\section{Contexto histórico: la historia y la geografía como canal de identidad nacional}

Después de la independencia de Colombia, las políticas educativas se orientaron a la alfabetización, los conocimientos matemáticos y la apropiación de una identidad nacional; por ello, las áreas de lenguaje, matemáticas, historia y geografía se convirtieron en ejes estructurales de la educación nacional. Desde entonces, durante el siglo xIx y hasta las primeras décadas del xx, la enseñanza de las ciencias sociales fue prioridad en la agenda del Estado.

La enseñanza de la geografía se convirtió en un eje central para generar identidad local, regional y nacional, por lo que se empezaron a desarrollar las primeras cartillas escolares donde se explicaban los elementos básicos del relieve colombiano, el clima, los recursos naturales y las características económicas y poblacionales de los departamentos y del país. La educación geográfica era positivista, descriptiva, enunciativa, básica, sin espacio para el debate o la argumentación; pero funcional para replicar el concepto de apropiación del territorio y la construcción de una identidad regional. Por su parte, la enseñanza de la historia se centró en la exaltación de héroes y hazañas de luchas libertadoras para justificar la emancipación de España y las bondades de esa separación. Se apeló a una enseñanza descriptiva de los hechos históricos, con un interés de construir identidad nacional, de justificar la existencia del Estado colombiano. Las descripciones históricas no se prestaron a matices: se era bueno si se era nacionalista y asesino si se era español; pero de igual forma se justificó como bueno todo lo traído por España: lengua, religión, principios, normas. Es decir, la cultura y la sociedad indígena se relegó y se avaló todo aquello que se deseaba que formara parte del proyecto nacional colombiano. Durante ciento veinte años la geografía y la historia se concibieron como áreas independientes, con elementos comunes, pero a fin de cuentas áreas de conocimiento propio, con fines particulares en la formación de nuevas generaciones.

Las reformas educativas de la década de 1930 determinaron que las ciencias sociales serían un programa de primero a quinto de primaria, con una subdivisión de áreas entre geografía, historia, democracia y urbanidad, con una intensidad de 6 horas a la semana. Para la educación secundaria se determinaron 4 horas semanales de historia y 3 de geografía de sexto a noveno grado. El currículo favoreció la elaboración de libros de texto para ambas áreas. Varias editoriales desarrollaron materiales de geografía de manera locacional, abordada por regiones. Los ejes temáticos por grados se distribuyeron de la siguiente manera: en sexto, geografía física de Colombia; en séptimo, geografía de América; en octavo, geografía de Europa, Asia, África y Oceanía; y en noveno, geografía económica con énfasis en Colombia. El currículo repetía procesos desde una visión tradicional y descriptiva.

\section{La integración}

En 1994 la Ley 115 de educación normativizó la integración de la geografía y la historia en una sola área, y redujo la intensidad horaria de 8 a 4 horas semanales. Además, se incluyeron las clases de democracia y todas las cátedras que se crearon a partir de la Constitución política de 1991 (entre ellas, las de afrocolombianidad, emprendimiento, las cátedras departamentales y regionales). La integración evidenció otra problemática y fue la supremacía de la historia sobre la geografía en la academia colombiana y en la formación escolar: tanto en la elaboración de los libros de texto como en los programas impartidos en la escuela, el $60 \%$ de los contenidos terminaron siendo históricos y el restante se distribuyó entre la formación política, la geografía y las demás cátedras. En eso se fue diluyendo la geografía en la escuela e inclusive se perdió su importancia y rol en la sociedad. Las nuevas generaciones en muchos casos desconocen qué es la geografía y cuáles son las funciones y acciones que despliega en su vínculo con la realidad.

El temor a la valoración y a la evaluación recrudece el problema. No hay claridad sobre los contenidos geográficos que se deben enseñar en la escuela. Se sigue oscilando entre la enseñanza descriptiva y la formación ciudadana. Los lineamientos del Ministerio de Educación para la enseñanza de las ciencias sociales expedidos en 2004 dan mayor claridad sobre qué enseñar en historia, pero ideas muy vagas frente a su pertinencia y contenidos para la enseñanza de la geografía.

Desde el 2016, y en el contexto del proceso de paz, varios historiadores y la comunidad histórica iniciaron un debate para revivir la cátedra de historia independiente de las ciencias sociales, como un compromiso por la paz, por conocer el pasado del país, explicar las razones del conflicto, entre otros aspectos. Sin embargo, los que lideraron el proceso desconocieron la enseñanza en el aula, los contenidos de ciencias sociales y, sobre todo, el entender que la integración fue el matrimonio de dos grandes áreas de conocimiento: la geografía y la historia. El 28 de diciembre de 2017 el Congreso de la República aprobó la creación de la cátedra de historia, y con ello la geografía siguió en el limbo, sin saber qué se enseña, las prioridades en la escuela y lo que se espera en los procesos de formación. Por otra parte, las pruebas nacionales han reducido la geografía al mínimo de evaluación.

\section{Formación para la enseñanza de la geografía: entre las políticas de calidad y la formación disciplinar}

La formación para los docentes que enseñan geografía implica desarrollar pensamiento espacial, articular la educación geográfica a contextos más cercanos y problematizar los más lejanos, formar ciudadanos, orquestar los estudios ambientales con relación a la apropiación 
antrópica del espacio geográfico e incluir otras ramas de la disciplina en la enseñanza escolar, como la económica, la política o los estudios de la población.

El Ministerio de Educación Nacional (MEN) enfatiza en que estas competencias se relacionan directamente con la práctica del maestro, los aprendizajes de los estudiantes y su formación integral. De manera complementaria, por medio de los Lineamientos de Calidad para las Licenciaturas en Educación, el Ministerio señala que en el caso particular de la formación inicial de maestros, los programas y su concreción en la propuesta curricular y el plan de estudios requieren de una necesaria y permanente articulación e interacción entre pedagogía, didáctica, disciplina e investigación para promover el aprendizaje de los estudiantes. El MEN da por sentado entonces que los ambientes de aprendizaje son el lugar donde el licenciado ejerce su práctica profesional y que es allí donde se concretan los aprendizajes de los maestros en formación.

Es esta una de las razones por las cuales la geografía escolar y la didáctica de la geografía deben tener mayor relevancia en ámbitos universitarios, proponiendo currículos en los que se consigne explícitamente el tipo de geografía que se quiere enseñar en las escuelas y qué procesos de enseñabilidad se buscan desarrollar en la formación de docentes. En la actualidad, la política educativa se orienta a definir los parámetros de calidad, tanto para los programas de formación como para las universidades que deseen acreditarse ${ }^{1}$. La Resolución 18583 del 2017 ajusta las características específicas de calidad de los programas de licenciatura para la obtención, renovación o modificación del registro calificado. Este documento contempla la posibilidad de que, además de las licenciaturas en ciencias sociales, exista la licenciatura en geografía. Así se abre el espacio, en el marco de la autonomía curricular de las instituciones, para que se incluyan los saberes específicos y disciplinares de esta área.

Del mismo modo, el decreto determina la importancia del saber didáctico para cada una de las disciplinas que estructuran la formación docente. Esto se refiere a la capacidad para aprehender y apropiar el contenido disciplinar como objeto de enseñanza. Con este fin, se hace pertinente conocer la manera en que las personas aprenden los contenidos y desarrollan habilidades concretas, reconocer dónde se encuentran las mayores dificultades del aprendizaje, así como utilizar estrategias y prácticas que permitan al estudiante resolver estas dificultades y evaluar los aprendizajes. Lo anterior implica una intersección entre los saberes didácticos y los contenidos disciplinares del área de desempeño del educador y sus prácticas pedagógicas, de forma que esté en capacidad de apropiar e investigar dichas prácticas y evaluar su impacto.

1 La acreditación de alta calidad es una directriz que permite el funcionamiento de un programa específico, en el que no solo se tienen en cuenta elementos curriculares, sino también otros aspectos ligados a la planta física, el perfil de los docentes, los resultados de pruebas externas y la formación del licenciado en diversos aspectos (académico, bienestar, investigación, entre otros).

\section{Las competencias y la didáctica de la geografía}

Si bien se discute la funcionalidad de las competencias con relación a la formación, estas se han permeado de políticas educativas y del desarrollo de microcurrículos. Sin embargo, a pesar de que la idea de competencia se encuentra inmersa en el lenguaje de los docentes, esta carece de aplicabilidad concreta en el proceso de enseñanza y menos aún es asimilada en las facultades de Educación, en parte porque las competencias se consideran una reproducción técnica del conocimiento y no la interiorización, el manejo y la resolución de situaciones problémicas de la cotidianidad. El MEN establece estas tres competencias básicas y fundamentales para la labor docente:

- Enseñar: para comprender, formular y usar la didáctica de las disciplinas con el propósito de favorecer los aprendizajes de los estudiantes.

- Formar: para re-conceptualizar y utilizar conocimientos pedagógicos que permitan crear ambientes educativos para el desarrollo de los estudiantes, del docente y de la comunidad.

- Evaluar: para hacer seguimiento y tomar decisiones sobre los procesos de formación, con el propósito de favorecer la autorregulación y de plantear acciones de mejora.

La ausencia de la formación en competencias profesionales en el marco universitario ha sido un problema latente hasta ahora, por lo que, a modo de alternativa, se realiza una propuesta de competencias focalizada para la asignatura de Didáctica de la geografía. Desde los microcurrículos ${ }^{2}$ se pueden desarrollar competencias del ser (afectiva), del saber (cognitiva) y del saber hacer (pedagógica). Estas competencias se definen así:

- Cognitivas: reconocer y diferenciar qué es y cómo se trabajan los saberes disciplinares, identificando así los instrumentos y las estrategias del saber ser, el saber conocer y el saber hacer, dentro de un contexto determinado.

- Afectivas: practicar la autorreflexión y metacognición para mejorar continuamente el desempeño, teniendo en cuenta determinados propósitos según el contexto de trabajo. Independientemente del modelo pedagógico que se trabaje en la institución universitaria es importante valorar el trabajo individual y cooperativo. 
- Pedagógicas: proceso de desempeño teórico-práctico con base en el desarrollo metacognitivo. El estudiante demuestra que internaliza el saber disciplinary tiene la capacidad de correlación teórico-práctica en diferentes ámbitos de formación.

Otro elemento fundamental es propiciar la investigación en el aula, puesto que es una manera de generar procesos autónomos de enseñanza-aprendizaje, aclarando que no se busca el desarrollo de la instrumentalización de la didáctica, sino un proceso de gestación de habilidades espaciales que le permitan al estudiante construir socialmente el espacio, al especificar la relación de las competencias en el marco de la enseñanza de la geografía. Finalmente, la aplicación de la "Trenza pedagógica”3 en diferentes asignaturas permite, además de un abordaje teórico per se, pensarse la articulación de los diferentes niveles de concreción curricular. Para el caso específico y en la búsqueda de la articulación de lo que se enseña en la universidad y lo que se busca que se enseñe en la escuela, se entiende la didáctica como un proceso de formación política del docente con miras a la reformulación de los procesos de enseñanza, rompiendo los imaginarios de instrumentalización, y la evaluación como proceso social que exige la transformación de la labor profesoral, articulando el conocimiento disciplinar universitario con las realidades de la escuela.

\section{La geografía escolar: la actual enseñanza en las aulas}

En 1994 el MEN expidió la Ley 115, mediante la cual se regularon los aspectos relacionados con la educación en el país. Uno de sus fines era "la adquisición y generación de los conocimientos científicos y técnicos más avanzados, humanísticos, históricos, sociales, geográficos y estéticos, mediante la apropiación de hábitos intelectuales adecuados para el desarrollo del saber" (Ley 115, art. 5, numeral 5, 1994). Lo anterior se interpreta como una preocupación por involucrar los conocimientos geográficos dentro de los procesos formativos de la población colombiana. No obstante, la misma ley centra el saber geográfico en temas referidos al conocimiento del medio físico, el uso de los recursos, la conservación del ambiente, la organización política del territorio y la observación de problemáticas sociales (sin especificar las espaciales), lo que evidencia el carácter descriptivo de las dinámicas espaciales y de su enseñanza. Dicha ley señaló entonces, para los niveles de educación básica y media en todo el país, la obligatoriedad del área de ciencias sociales integrando dentro de ella el estudio de la historia, la geografía, la Constitución política y la democracia.

\footnotetext{
3 Nombre que la doctora Luz Stella García (docente de la Universidad del Tolima) ha establecido para la unión de los conceptos de currículo, didáctica y evaluación en pro de la mejora del proceso de enseñanza-aprendizaje.
}

Desde esta perspectiva, con la Resolución 2343 de 1996 se intentó regular el diseño de un currículo integrado para las ciencias sociales a partir de los indicadores de logros curriculares que, para el caso de la enseñanza de la geografía, se concretaron en los contenidos y procesos para los diferentes grados de escolaridad que se presentan en la tabla 1.

Tabla 1. Contenidos y procesos geográficos referidos en los logros curriculares

\begin{tabular}{|c|c|c|c|c|c|}
\hline & Preescolar & $\begin{array}{c}\text { Primero, } \\
\text { segundo, } \\
\text { tercero }\end{array}$ & $\begin{array}{c}\text { Cuarto, quinto, } \\
\text { sexto }\end{array}$ & $\begin{array}{c}\text { Séptimo, } \\
\text { octavo, noveno }\end{array}$ & $\begin{array}{l}\text { Décimo, } \\
\text { undécimo }\end{array}$ \\
\hline 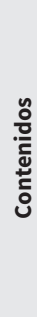 & $\begin{array}{l}\text { Ambiente, } \\
\text { mundo } \\
\text { físico, } \\
\text { entorno }\end{array}$ & $\begin{array}{l}\text { Elementos } \\
\text { geográficos, } \\
\text { paisaje cultu- } \\
\text { ral y natural, } \\
\text { recursos natu- } \\
\text { rales, modelos } \\
\text { de representa- } \\
\text { ción, nociones } \\
\text { de organiza- } \\
\text { ción territorial }\end{array}$ & $\begin{array}{l}\text { Cartografía, } \\
\text { la Tierra, el } \\
\text { sistema solar, } \\
\text { elementos } \\
\text { de la superfi- } \\
\text { cie terrestre, } \\
\text { actividades } \\
\text { productivas, } \\
\text { problemáticas } \\
\text { ambientales }\end{array}$ & $\begin{array}{l}\text { Fenómenos } \\
\text { sociales espa- } \\
\text { cial y tem- } \\
\text { poralmente } \\
\text { situados }\end{array}$ & $\begin{array}{l}\text { Problemáticas } \\
\text { sociales, cul- } \\
\text { turales, polí- } \\
\text { ticas, étnicas, } \\
\text { económicas y } \\
\text { ambientales }\end{array}$ \\
\hline 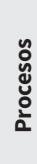 & $\begin{array}{l}\text { Orienta- } \\
\text { ción, obser- } \\
\text { vación, } \\
\text { ubicación }\end{array}$ & $\begin{array}{l}\text { Identifi- } \\
\text { car, clasifi- } \\
\text { car, valorar, } \\
\text { construir, } \\
\text { organizar. }\end{array}$ & $\begin{array}{l}\text { Identificar, } \\
\text { reconocer, } \\
\text { relacionar }\end{array}$ & Comparación & $\begin{array}{l}\text { Plantear } \\
\text { criterios }\end{array}$ \\
\hline
\end{tabular}

Fuente: elaboración propia a partir de la Resolución 2343 (1996).

En este caso, se evidencia una preocupación por identificar, clasificar y comparar los elementos físicos propios del paisaje, la descripción de las actividades humanas productivas y las problemáticas derivadas de ellas sin llegar a generar procesos de análisis de ellas (solo en los grados décimo y once). Sumado a lo anterior, se puede vislumbrar cómo los contenidos geográficos tienen mayor relevancia en los primeros grados de la educación básica, mientras que de séptimo a once se restringe a temáticas referentes a la ubicación espacial de los fenómenos sociales en función de aspectos históricos y ambientales del país.

Por su parte, los Lineamientos Curriculares se concibieron como una propuesta "abierta, flexible, integrada y en espiral" (MEN, 2002), implementada a partir de ejes generadores (agrupación de temáticas), preguntas orientadoras (promoción de la investigación) y ámbitos conceptuales (agrupación de conceptos), que en su conjunto buscaban desarrollar las competencias propias de los científicos sociales. Esta propuesta, aunque se estructuró con base en la concepción de integración formulada desde la Ley 115 , enmarca una perspectiva histórica notoria, como se evidencia en la enunciación de las pautas de secuencia para el área, cuyos referentes se centran en lo cognitivo, las vivencias, las experiencias, el manejo conceptual, la perspectiva temporal y el tiempo histórico. A lo anterior, se suma el análisis de los aspectos espaciales propuestos dentro de los ejes generadores, que se detallan de la siguiente manera: 1) mujeres y hom- 
bres como guardianes y beneficiarios de la madre tierra, 2) la necesidad de buscar desarrollos económicos sostenibles que permitan preservar la dignidad humana y 3) nuestro planeta como un espacio de interacciones cambiantes que nos posibilita y limita. Estos ejes privilegian temáticas ambientales, el uso de los recursos y el desarrollo económico, pero dejan de lado la enseñanza de las habilidades propias del pensamiento espacial y los contenidos que permiten el análisis del dinamismo y complejidad de los problemas propios del espacio geográfico.

Respecto a los Estándares Básicos (MEN, 2006), estos se centran en señalar "[...] aquello que todos los estudiantes del país, independientemente de la región en la que se encuentren, deben saber y saber hacer una vez finalizado su paso por un grupo de grados" (p. 113), buscando responder a una integración curricular desde el desarrollo de las competencias científicas a partir de tres ejes articuladores: 1) aproximación al conocimiento científico-social, 2) manejo de los conocimientos propios de las ciencias sociales a partir de tres grandes relaciones (historia-cultura, espacio-medioambiente y ética-política), y 3) desarrollo de compromisos personales y sociales. En consecuencia, los contenidos y procesos geográficos referenciados en los Estándares Básicos se resumen en la tabla 2.

Tabla 2. Contenidos y procesos geográficos referidos en los Estándares Básicos

\begin{tabular}{|c|c|c|c|c|c|}
\hline & $\begin{array}{l}\text { Primero, } \\
\text { segundo, } \\
\text { tercero }\end{array}$ & $\begin{array}{l}\text { Cuarto, } \\
\text { quinto }\end{array}$ & $\begin{array}{l}\text { Sexto, } \\
\text { séptimo }\end{array}$ & $\begin{array}{l}\text { Octavo, } \\
\text { noveno }\end{array}$ & $\begin{array}{l}\text { Décimo, } \\
\text { undécimo, } \\
\text { once }\end{array}$ \\
\hline 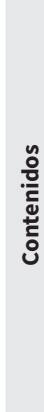 & $\begin{array}{l}\text { Ubicación } \\
\text { espacial, refe- } \\
\text { rentes y repre- } \\
\text { sentaciones } \\
\text { espaciales, } \\
\text { formas del } \\
\text { paisaje natu- } \\
\text { ral y cultural, } \\
\text { actividades } \\
\text { económi- } \\
\text { cas, recursos } \\
\text { naturales }\end{array}$ & $\begin{array}{l}\text { Ubicación } \\
\text { espacial, refe- } \\
\text { rentes y repre- } \\
\text { sentaciones } \\
\text { espaciales, } \\
\text { regiones, acti- } \\
\text { vidades eco- } \\
\text { nómicas, uso } \\
\text { de la tierra }\end{array}$ & $\begin{array}{l}\text { El planeta } \\
\text { Tierra, carto- } \\
\text { grafía, husos } \\
\text { horarios, } \\
\text { características } \\
\text { físicas y climá- } \\
\text { ticas del espa- } \\
\text { cio, organiza- } \\
\text { ción espacial } \\
\text { y económica } \\
\text { de las culturas } \\
\text { a través del } \\
\text { tiempo }\end{array}$ & $\begin{array}{c}\text { Ecosistemas, } \\
\text { ambiente y } \\
\text { economía, } \\
\text { historia eco- } \\
\text { nómica de } \\
\text { Colombia, } \\
\text { migración } \\
\text { y desplaza- } \\
\text { miento }\end{array}$ & $\begin{array}{l}\text { Modelos eco- } \\
\text { nómicos en } \\
\text { Colombia y el } \\
\text { mundo, desa- } \\
\text { rrollo econó- } \\
\text { mico, rela- } \\
\text { ciones eco- } \\
\text { nómicas y su } \\
\text { organización, } \\
\text { globalización, } \\
\text { concentración } \\
\text { urbana }\end{array}$ \\
\hline : & $\begin{array}{l}\text { Reconoci- } \\
\text { miento de } \\
\text { la interac- } \\
\text { ción entre el } \\
\text { ser humano } \\
\text { y el paisaje } \\
\text { a partir de } \\
\text { las acciones } \\
\text { económicas }\end{array}$ & $\begin{array}{l}\text { Reconoci- } \\
\text { miento de las } \\
\text { característi- } \\
\text { cas físicas y } \\
\text { culturales del } \\
\text { entorno y sus } \\
\text { consecuencias }\end{array}$ & $\begin{array}{l}\text { Análisis sobre } \\
\text { producción, } \\
\text { transforma- } \\
\text { ción y dis- } \\
\text { tribución de } \\
\text { recursos, bie- } \\
\text { nes y servicios } \\
\text { de acuerdo } \\
\text { con las carac- } \\
\text { terísticas } \\
\text { físicas del } \\
\text { entorno }\end{array}$ & $\begin{array}{l}\text { Análisis de } \\
\text { la interac- } \\
\text { ción entre el } \\
\text { espacio geo- } \\
\text { gráfico y el } \\
\text { ser humano } \\
\text { evaluando los } \\
\text { avances y limi- } \\
\text { taciones de } \\
\text { esta relación }\end{array}$ & $\begin{array}{l}\text { Identificación } \\
\text { y postura de } \\
\text { las causas y } \\
\text { consecuen- } \\
\text { cias políticas, } \\
\text { económicas, } \\
\text { sociales y } \\
\text { ambientales } \\
\text { de la aplica- } \\
\text { ción de las } \\
\text { diferentes teo- } \\
\text { rías y modelos } \\
\text { económicos }\end{array}$ \\
\hline
\end{tabular}

Fuente: elaboración propia a partir de los Estándares Básicos de Competencias en Ciencias Sociales (MEN, 2006).
La anterior propuesta se centra en la descripción de las características naturales y culturales del paisaje (con un alto componente histórico evidenciado en la interpretación de los cambios espaciales en las diferentes culturas) y el análisis de las dinámicas económicas humanas desarrolladas por las sociedades en el espacio. De esta manera, no se observa una progresión clara en el desarrollo de los procesos y habilidades para el análisis espacial ni una secuencia temática estructurada.

En el 2016 se presentaron los Derechos Básicos de Aprendizaje (DBA), que, en coherencia con los Lineamientos Curriculares y los Estándares Básicos, buscaban generar una secuencia en el aprendizaje a partir de la integración de conocimientos, habilidades y actitudes. Se consideran "estructurantes en tanto expresan las unidades básicas y fundamentales sobre las cuales se puede edificar el desarrollo futuro del individuo" (MEN, 2016, p. 6). Para el área de ciencias sociales, los DBA quedaron agrupados en cuatro categorías organizadoras: espacialidad, temporalidad, culturalidad e institucionalidad y derechos humanos. En la tabla 3 se sintetiza la progresión de los DBA para los diferentes grados con respecto a la espacialidad.

Tabla 3. Derechos Básicos de Aprendizaje para la categoría de espacialidad

Primaria
Primero: 1 . Ubicación espacial a partir
del cuerpo y los puntos cardinales.
2. Descripción de las características del
paisaje geográfico (lugar donde vive).

Segundo: 1. Comprensión de los cambios del paisaje por acciones humanas. 2. Reconocimiento de los puntos cardinales para la orientación y el desplazamiento.

Tercero: 1 . Comprensión de la importancia de océanos y mares en la economía de los pueblos costeros. 2. Relación de las características biogeográficas con las actividades económicas.

Cuarto: 1 . Comprensión de la importancia de los límites geográficos y las fronteras en la organización de los territorios. 2. Diferenciación a las características del medio urbano y el medio rural (concentración de la población y uso del suelo).

Quinto: 1. Comprensión de la organización territorial por regiones. 2. Comprensión de las ventajas de la posición geográfica y astronómica de Colombia en relación con la economía.

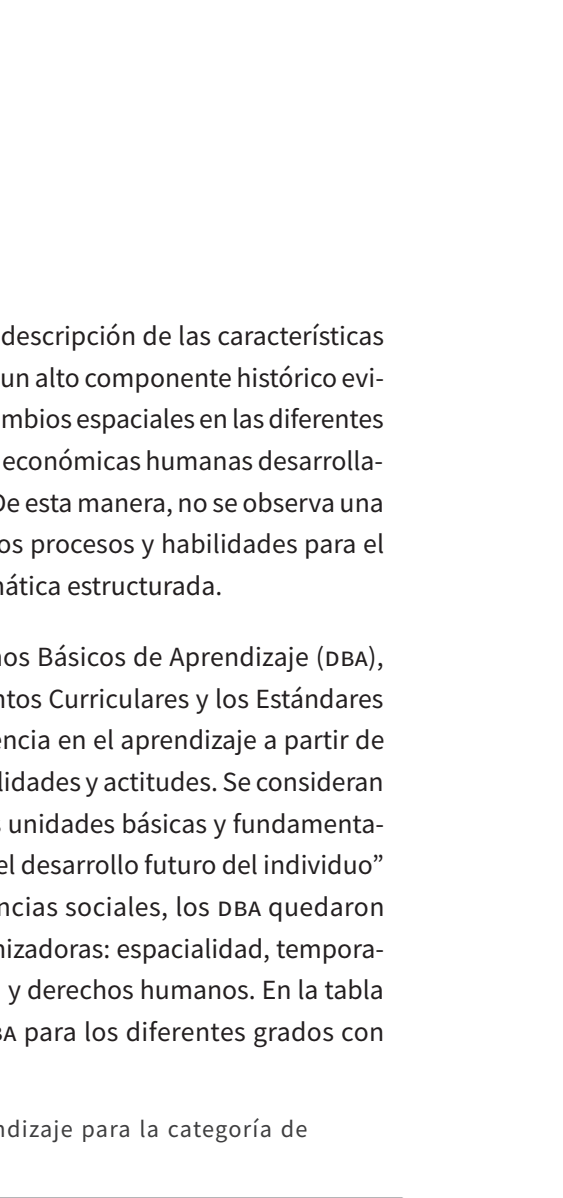

Sexto: 1. Comprensión de las teorías sobre el origen del universo. 2. Comprensión de los cambios del relieve terrestre y su incidencia en las comunidades.

Séptimo: 1 . Comprensión de los cambios de las representaciones del mundo. 2. Interpretación de las relaciones entre el crecimiento de la población, el desarrollo urbano y las problemáticas sociales.

Octavo: 1. Evaluación de la influencia de los procesos de cooperación económica y política entre los Estados.

2. Comprensión del fenómeno migratorio en el mundo y sus consecuencias.

Noveno: 1. Análisis de la situación ambiental de los geosistemas de Colombia y las problemáticas derivadas de su explotación. 2. Comprensión de las consecuencias de los procesos migratorios en Colombia.

Décimo: 1. Análisis de los conflictos en el territorio colombiano relacionados con la degradación ambiental, el desarrollo económico y la inestabilidad política.

Undécimo: 1 . Análisis de la supervivencia de la humanidad a partir de la protección del ambiente.

Fuente: elaboración propia a partir de los DBA (MEN, 2016). 
Los DBA están correlacionados con las temáticas orientadas en los Logros Curriculares de 1996, en este caso reducidos a dos los derroteros por grado para el estudio de la geografía. En el caso de primaria se da relevancia al desarrollo de la orientación, la identificación de las características del paisaje, su relación con las actividades económicas, y la descripción de los entornos rurales y urbanos. Se resalta la incorporación del estudio de los mares y los océanos en las dinámicas económicas de los pueblos costeros. En secundaria es preponderante el estudio de los elementos del universo, la descripción del relieve terrestre y la interpretación de las representaciones espaciales, además de las temáticas relacionadas con el crecimiento de la población y sus consecuencias en la organización de los centros urbanos.

Hasta aquí es claro cómo desde la integración de las ciencias sociales a partir de la Ley 115 de 1994 la enseñanza de la geografía ha estado dirigida al desarrollo de la orientación, la lectura de representaciones espaciales, la descripción de los elementos naturales y culturales del paisaje, la caracterización de las regiones, la delimitación territorial, la caracterización de las actividades económicas, entre otras temáticas que, a pesar de la idea de integración curricular, han estado relegadas ante el estudio de los aspectos históricos. De allí que sea indispensable analizar la pertinencia de los contenidos diseñados para el estudio del espacio geográfico, además de la secuencia de los procesos, que permite el análisis espacial en relación con las dinámicas sociales.

\section{¿Qué enseñar?}

Frente a la falta de un lugar definido para los procesos de la enseñanza de la geografía en las ciencias sociales escolares, evidenciada en la preponderancia de la historia y la poca claridad en la secuencia de contenidos y los procesos para el análisis geográfico, es importante establecer la pertinencia de algunos de los contenidos y procesos a partir de las reflexiones didácticas resultantes de investigaciones de diversos grupos en Iberoamérica, de las reflexiones nacientes en el grupo Geopaideia y de la misma práctica docente.

El qué enseñar parte de la necesidad de generar una integración de las habilidades conceptuales, procedimentales y actitudinales, que más allá de lo propuesto hasta el momento con la integración de la historia, la geografía, la Constitución política y la democracia en el área de ciencias sociales, propende por la articulación de conceptos y procedimientos geográficos para la resolución de problemas propios del espacio geográfico que permitan una mejor lectura y análisis de la organización espacial en relación con la realidad cotidiana de los estudiantes. En este sentido, a partir de lo expuesto por Castellar (2014) para el desarrollo del pensamiento espacial y el análisis geográfico, se hace pertinente tener en cuenta el mundo de la vida en la generación de contenidos y estrategias de enseñanza.
Los procesos didácticos referentes a la geografía, según Souto (1999), implican la formulación de conceptos, hechos y estructuras relacionadas entre sí con base en problemáticas socioespaciales, cuyo conocimiento permite el análisis de lugares diferenciados y delimitados a partir de conceptos específicos, con el propósito de convertirse en saberes útiles para el estudiante. En este contexto, de acuerdo con Souto (2017), la preocupación por el análisis del Espacio geográfico como categoría para la enseñanza de la geografía, implica tener como referentes subcategorías como las de Espacio percibido, Espacio vivido y Espacio concebido, de manera contextualizada a partir de problemáticas sociales visibles para los estudiantes. No obstante, es importante resaltar que la elección de los conceptos asociados con el análisis del espacio geográfico parte también del enfoque epistemológico que el docente maneje y de su interés disciplinar.

Así, por ejemplo, para Blanco (2009) son primordiales los conceptos de paisaje, región, espacio, lugar y territorio; sin embargo, cada uno de estos puede abordarse desde diferentes enfoques teniendo en cuenta la escuela geográfica y pedagógica que oriente la reflexión docente. Independientemente de la orientación epistemológica del educador, la relevancia de los conceptos tiene que ver con el diálogo que estos establecen con las problemáticas socioespaciales del contexto del estudiante. Desde esta premisa son varias las propuestas que buscan generar una secuencia didáctica para la enseñanza de la geografía. Este es el caso de lo planteado por Rodríguez de Moreno (2000 y 2010), quien propone una secuencia para primaria fundamentada en conceptos propios de la geografía y una para secundaria desde la perspectiva de las escuelas geográficas.

Tabla 4. Secuencia temática y conceptual

\begin{tabular}{|c|c|c|}
\hline Grado & Eje temático & Concepto/escuela geográfica \\
\hline 1 & La vivienda & Relaciones espaciales \\
\hline 2 & Representación topográfica & $\begin{array}{l}\text { Tamaño, proximidad, altura, } \\
\text { distancia, causalidad simple }\end{array}$ \\
\hline 3 & Representación geométrica & $\begin{array}{l}\text { Orientación, localización, escala y } \\
\text { convenciones }\end{array}$ \\
\hline 4 & Espacio geográfico & $\begin{array}{l}\text { Sociedad, medio, entorno, paisaje, } \\
\text { ambiente, espacio geográfico }\end{array}$ \\
\hline 5 & La ciudad & Espacio geográfico urbano \\
\hline 6 & $\begin{array}{l}\text { Relieve, geomorfología, clima, } \\
\text { vegetación }\end{array}$ & Geografía física \\
\hline 7 y 8 & $\begin{array}{l}\text { Poblamiento, densidad, grupos } \\
\text { étnicos, asentamientos }\end{array}$ & Geografía humana \\
\hline 9 & $\begin{array}{l}\text { Imagen de la ciudad o del entorno } \\
\text { cercano }\end{array}$ & Geografía de la percepción \\
\hline 9 & $\begin{array}{l}\text { Densidad y distribución de la } \\
\text { estructura espacial }\end{array}$ & Enfoque positivista \\
\hline 10 & $\begin{array}{l}\text { Problemáticas políticas, económicas, } \\
\text { sociales, culturales }\end{array}$ & Enfoque hermenéutico \\
\hline 11 & Problemas socioespaciales & $\begin{array}{l}\text { Enfoque sociocrítico-geografía } \\
\text { radical }\end{array}$ \\
\hline
\end{tabular}

Fuente: Rodríguez de Moreno (2000 y 2010). 
Finalmente, como parte de la elección de los contenidos y procesos geográficos por enseñar, Caballero (2000) propone algunos criterios de selección, como la integración disciplinar, la recurrencia, la relevancia, la proximidad, la complejidad y el desarrollo de la capacidad crítica. Así, se entiende la importancia del contexto y las problemáticas socioespaciales como primer paso para la elección de los contenidos y procesos espaciales, iluminadas por el enfoque epistemológico que permite establecer, además de las habilidades por desarrollar, la pertinencia de la temática y el contexto.

\section{¿Cómo enseñarlo?}

En adición al qué enseñar, se genera la reflexión por el cómo enseñar los elementos que permitan el conocimiento y análisis del espacio geográfico. A partir de las investigaciones sobre la didáctica de la geografía, se presentan las siguientes generalidades: en primer lugar, se hace necesario construir una secuencia de contenidos y procesos que permitan conocer y analizar las realidades locales, nacionales y mundiales de una manera contextualizada, que conduzcan al desarrollo de habilidades propias del pensamiento espacial. De allí que se retomen propuestas como la de Franco, Montañez, Rodríguez de Moreno y Cárdenas de Torres (2000), desde la cual se involucran para cada grado, de primero a noveno, el horizonte conceptual, los objetivos de enseñanza y los conceptos y contenidos por desarrollar en la enseñanza de la geografía.

En segundo lugar, es importante conocer la manera como los estudiantes comprenden el saber teórico y lo relacionan con el saber práctico desde el inicio hasta el final del proceso educativo. En este sentido, es preciso indagar acerca de los saberes preexistentes y las expectativas de los estudiantes en relación con el contexto social, con el propósito de evidenciar los saberes previos e identificar dificultades y potencialidades en el proceso de aprendizaje. A partir de lo anterior se propone generar preguntas que impliquen un método de trabajo para resolverlas. Muchas de las reflexiones didácticas frente a la enseñanza de la geografía (CabaIlero, 2000; Rodríguez de Moreno, 2000; Sebastiá y Tonda, 2017; Souto, 1999) propenden por una didáctica basada en un método investigativo desde el cual se plantea un objeto de indagación, y es tarea del estudiante resolverla y socializarla bajo la orientación del docente.

En tercer lugar, tenemos la combinación de diferentes estrategias para el aprendizaje, las cuales se relacionan con el reconocimiento de las ideas previas de los estudiantes, la generación de un clima de aula que facilite el aprendizaje, la definición de objetivos por parte del docente, la consecución de trabajos individuales y grupales que faciliten la construcción colectiva del conocimiento, el uso de diferentes fuentes de información, la utilización de recursos variados, las salidas de campo como posibilidad de vivenciar los aprendizajes $y$, sobre todo, el sentido que se da al aprendizaje de diferentes contenidos y procesos.
En cuarto lugar, se tiene en cuenta el desarrollo de habilidades de pensamiento que permitan la explicación de las dinámicas del espacio geográfico. Rodríguez, E., Pérez, G. y Rodríguez, L. (2003) recalcan la importancia de dos habilidades primordiales: la observación y la descripción. La primera, orientada al contacto sensorial con la realidad espacial para el cuestionamiento, la comparación y la retrospectiva; la segunda, enfocada a contrastar, localizar y desarrollar la proporcionalidad y la densidad, y relacionar elementos temporales como el ritmo, el cambio, la posición y la velocidad.

Finalmente, sin pretender dar por acabada la discusión del cómo enseñar, se resalta el papel del lenguaje en los procesos de aprendizaje. Desde el siglo pasado, con los giros lingüístico y espacial, se ha dado importancia a otros tipos de lenguaje, además del verbal, para comprender la realidad social. Para el caso de la enseñanza de la geografía, es más que válido el uso de diferentes lenguajes para resolver las preguntas propuestas por los estudiantes y dotar de sentido al proceso de aprendizaje. De allí que propuestas como la de Souto (1999 y 2017) tengan en cuenta lo cartográfico, lo icónico, lo estadístico y lo verbal para atender una secuencia didáctica. De igual manera, investigaciones vigentes del grupo Geopaideia han centrado su interés en el desarrollo de habilidades espaciales y el análisis del espacio geográfico a partir de escenarios como la literatura o la fotografía, no solo como fuentes de información o documentación, sino como posibilidades didácticas.

Lo anterior nos permite generar una reflexión en torno a la necesidad de establecer una secuencia de aprendizaje, buscando dar un lugar preponderante al estudio del espacio geográfico, además de hacer frente a la falta de unificación de un currículo pertinente para la enseñanza de la geografía y al reduccionismo dado a los contenidos geográficos centrados en la enunciación de los aspectos del paisaje, la caracterización de las problemáticas ambientales y el estudio de las dinámicas económicas.

\section{Una propuesta curricular en geografía}

Un debate que hemos sugerido es la necesidad de definir el qué y para qué enseñar geografía en la escuela. El requisito de habilidades y competencias que necesita una persona para hacer el tránsito a la educación superior ha difuminado la importancia del pensamiento espacial en la formación de ciudadanos críticos reconocedores de su entorno. Varios académicos señalan que es necesario no sobrecargar a los estudiantes con autores complejos o con dinámicas como construir mapas por curvas de nivel cuando la prioridad es que aprendan a utilizar las representaciones cartográficas y a orientarse a través de ellas. A partir de esta premisa, la propuesta de Rodríguez Pizzinato (2007) presenta la siguiente estructura conceptual para la enseñanza de la geografía: 
Gráfico 1. Estructura conceptual de la geografía escolar

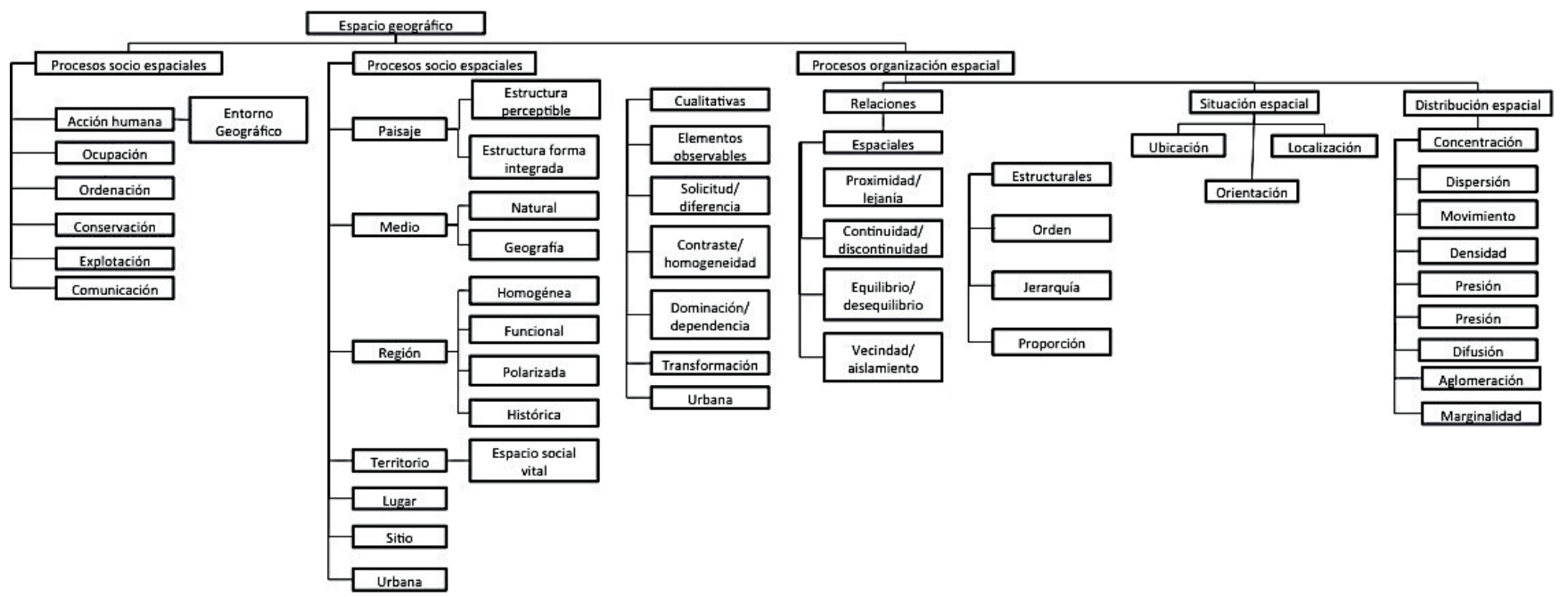

Fuente: Rodríguez (2007).

Debe mantenerse la integración de la geografía con la historia y la formación ciudadana. El reto es superar la visión tradicional que la mayoría de instituciones educativas sostiene. Lo que se enseña hasta hoy, en primero (la familia y el entorno), segundo (el barrio y la localidad), tercero (la ciudad y el departamento), cuarto (relieve, clima y aspectos físicos de Colombia) y quinto (geografía de la población y económica de Colombia), desfavorece la construcción de un pensamiento espacial holístico, dificulta la implementación de un currículo nacional e incentiva la enseñanza locacional y descriptiva. Es por ello que la propuesta para la enseñanza de la geografía la incorporamos para los grados tercero, cuarto y quinto, para los dos primeros se considera pertinente la formación ciudadana a partir del entorno local y la identificación de los elementos del espacio geográfico cotidiano.
Tabla 5. Secuencia temática y conceptual para grados tercero, cuarto y quinto

\begin{tabular}{|l|c|c|c|c|c|}
\hline Grados & \multicolumn{5}{|c|}{ Ejes conceptuales } \\
\hline Tercero & $\begin{array}{c}\text { Manejo de } \\
\text { distancias }\end{array}$ & $\begin{array}{c}\text { Uso de } \\
\text { mapasy } \\
\text { planos }\end{array}$ & $\begin{array}{c}\text { Elaboración } \\
\text { de mapas }\end{array}$ & $\begin{array}{c}\text { El concepto } \\
\text { de frontera }\end{array}$ & $\begin{array}{c}\text { Los } \\
\text { conceptos } \\
\text { geográficos }\end{array}$ \\
\hline Cuarto & $\begin{array}{c}\text { El espacio } \\
\text { geográfico }\end{array}$ & $\begin{array}{c}\text { Lo urbano y } \\
\text { lo rural }\end{array}$ & El paisaje & El relieve & El clima \\
\hline Quinto & $\begin{array}{c}\text { El espacio } \\
\text { habitado }\end{array}$ & $\begin{array}{c}\text { El uso del } \\
\text { suelo }\end{array}$ & $\begin{array}{c}\text { Las formas y } \\
\text { funciones de } \\
\text { la ciudad }\end{array}$ & $\begin{array}{c}\text { Las formas y } \\
\text { lanciones } \\
\text { de los } \\
\text { espacios } \\
\text { rurales }\end{array}$ & $\begin{array}{c}\text { Conceptos } \\
\text { básicos de la } \\
\text { demografía }\end{array}$ \\
\hline
\end{tabular}

Fuente: elaboración de los autores.

En secundaria, no ha existido un criterio único sobre qué enseñar en geografía. En varias instituciones se sigue aplicando la enseñanza de la geografía locacional, como se hacía antes de 1994. 
Tabla 6. Secuencia temática y conceptual para secundaria.

\begin{tabular}{|c|c|c|c|c|c|}
\hline Grado & \multicolumn{5}{|c|}{ Ejes conceptuales } \\
\hline Sexto & Cartografía & Relieve & $\begin{array}{l}\text { Geomorfo- } \\
\text { logía }\end{array}$ & Clima & $\begin{array}{l}\text { Proble- } \\
\text { máticas } \\
\text { ambientales }\end{array}$ \\
\hline Séptimo & Demografía & $\begin{array}{l}\text { Elementos de } \\
\text { la geografía } \\
\text { poblacional }\end{array}$ & $\begin{array}{l}\text { Estructuras } \\
\text { demográficas }\end{array}$ & $\begin{array}{l}\text { Caracte- } \\
\text { rísticas } \\
\text { culturales }\end{array}$ & $\begin{array}{l}\text { Las } \\
\text { migraciones }\end{array}$ \\
\hline Octavo & $\begin{array}{l}\text { Estructura } \\
\text { y tipos de } \\
\text { Estado }\end{array}$ & $\begin{array}{l}\text { La organi- } \\
\text { zación del } \\
\text { territorio }\end{array}$ & $\begin{array}{l}\text { La organiza- } \\
\text { ción mundial }\end{array}$ & $\begin{array}{l}\text { Los espa- } \\
\text { cios urba- } \\
\text { nosy } \\
\text { rurales }\end{array}$ & $\begin{array}{l}\text { El concepto } \\
\text { de ciudad }\end{array}$ \\
\hline Noveno & $\begin{array}{l}\text { Los sectores } \\
\text { económicos }\end{array}$ & $\begin{array}{l}\text { Las acti- } \\
\text { vidades } \\
\text { económicas }\end{array}$ & $\begin{array}{l}\text { La } \\
\text { globalización }\end{array}$ & $\begin{array}{l}\text { Redes } \\
\text { y flujos } \\
\text { comerciales }\end{array}$ & $\begin{array}{l}\text { Los con- } \\
\text { flictos y los } \\
\text { recursos }\end{array}$ \\
\hline Décimo & $\begin{array}{l}\text { Espacio } \\
\text { rural }\end{array}$ & $\begin{array}{l}\text { Actividades } \\
\text { agrarias }\end{array}$ & $\begin{array}{l}\text { Espacio } \\
\text { urbano }\end{array}$ & $\begin{array}{l}\text { Actividades } \\
\text { urbanas }\end{array}$ & $\begin{array}{l}\text { Los espa- } \\
\text { cios } \\
\text { suburbanos }\end{array}$ \\
\hline Undécimo & $\begin{array}{l}\text { La } \\
\text { geopolítica }\end{array}$ & $\begin{array}{l}\text { Podery } \\
\text { territorio }\end{array}$ & $\begin{array}{l}\text { Formas de } \\
\text { participación } \\
\text { y representa- } \\
\text { ción }\end{array}$ & & \\
\hline
\end{tabular}

Fuente: elaboración propia.

\section{Los contenidos y los materiales educativos}

En el xvı Congreso Colombiano de Geografía del año 2000, los profesores Franco, Montañez, Rodríguez y Torres presentaron un artículo llamado "Propuesta para la enseñanza de la educación geográfica", en el cual plantearon unos contenidos locacionales desde los conceptos y las redes conceptuales. Hoy el panorama y las necesidades son otras, por eso el papel que desempeñan los textos escolares es fundamental.

Tradicionalmente, los textos diseñados por las editoriales se convierten en el principal recurso para la enseñanza de la geografía. Los textos enfrentan varias dificultades, una de ellas refiere a la falta de una secuencia temática, lo que conduce a la elaboración de propuestas basadas en criterios particulares de los editores. Otra dificultad se relaciona con la manera como se presenta la información geográfica y el enfoque que se le da, lo que genera vacíos didácticos y centra su interés en dotar al docente de herramientas teóricas sin tener presentes de manera clara los procesos de aprendizaje de los estudiantes. Sumado a lo anterior, la enseñanza de la geografía se reduce a un $20 \%$ o $30 \%$ de la estructura total de los libros de ciencias sociales, ya que se le da mayor importancia a los temas relacionados con la historia, sin responder realmente a los fines de la integración curricular propuesta por el Ministerio de Educación desde 1994.
Sin embargo, en los últimos años se resaltan propuestas editoriales que buscan desarrollar contenidos centrados en el aprendizaje de los estudiantes, no solo desde los aspectos visuales o de lenguaje propios de cada edad, sino también con base en actividades contextualizadas y significativas. Además, se ha evidenciado una preocupación por la renovación conceptual y pedagógica, que en algunos casos ha propiciado la reelaboración de los textos escolares.

Ante estas premisas se puede indicar que el uso del libro de texto parte de la visión epistemológica del docente y su intencionalidad didáctica. En muchos casos la enseñanza de la geografía queda relegada a temas y actividades propuestas en los libros de texto. En otros casos, el libro se convierte en una de las variadas fuentes que pueden utilizar los estudiantes para organizar y analizar los contenidos y problemáticas socioespaciales. De allí la importancia de involucrar dentro de los procesos de enseñanza y aprendizaje las unidades didácticas, con las cuales se proponen objetivos, actividades, recursos y estrategias de evaluación de los temas relacionados con el estudio del espacio geográfico. Las unidades didácticas pueden responder de manera más efectiva a las necesidades de aprendizaje de los estudiantes y a los intereses de enseñanza de los docentes, ya que son confeccionadas a partir de las realidades próximas y cotidianas.

\section{Reflexiones finales}

Las políticas educativas para las ciencias sociales en Colombia, desde el siglo xIx, se han orientado a responder a un modelo de nación desde el reconocimiento de aquellos elementos representativos, que desde un enfoque temporal y espacial exaltan la identidad del país. En este contexto, la enseñanza de la historia y de la geografía ha privilegiado la descripción y enunciación de eventos y hazañas de sujetos particulares y las características del paisaje, por encima del debate y la argumentación de los problemas sociales.

Respecto a la enseñanza de la geografía, se evidencia un detrimento tanto en sus contenidos como en su intensidad horaria dentro del currículo escolar, sumado a la prevalencia de los estudios históricos, que relegan a la geografía al estudio del espacio como contenedor y a la descripción de los aspectos morfológicos a nivel físico, sin generar relaciones claras ni un análisis del espacio geográfico desde la apropiación de sus categorías, el desarrollo de habilidades espaciales y la generación de prácticas que permitan mejorar la calidad de vida de las sociedades.

Desde lo legislativo, tanto en el contexto escolar como en los programas de formación de docentes, se han viabilizado propuestas que buscan la integración de las ciencias sociales y el fomento de la calidad educativa; sin embargo, estas políticas se perciben ambiguas y con un mínimo alcance y materialización en los programas de estudio y en las aulas de clase. Ante este panorama, grupos de investigación relacionados con la enseñanza de la geografía han propuesto, desde la praxis educativa, 
alternativas para la formación de docentes y para la consecución de una geografía escolar pertinente, contextualizada y, sobre todo, útil a las necesidades del estudiantado y de la sociedad.

En Colombia estamos ante una geografía escolar que desaparece, por lo que debe ser compromiso de los grupos de investigación, de la academia y del Gobierno luchar por la recuperación de la educación geográfica. No obstante, lo primordial es definir qué enseñar y para qué hacerlo, establecer un programa curriculary unos lineamientos por grados de primero a undécimo que vayan de la mano con una estrategia que se acompañe de reformas necesarias en la formación docente, en el uso de los materiales de texto y en los procesos de evaluación de la escuela y del Estado.

La geografía escolar debe posibilitar el análisis de las relaciones espaciales, temporales y sociales del espacio geográfico, problematizar las realidades espaciales y generar alternativas de solución desde el desarrollo del saber, el hacer y el ser. Debe rebasar la tradicional enseñanza basada en la enunciación de los elementos del paisaje o la homogeneización de las características culturales de una región o nación. Para ello se plantea, desde la formación docente, el desarrollo de competencias cognitivas, afectivas y pedagógicas que aporten a la reflexión y práctica de la didáctica de la geografía; desde la práctica escolar, una propuesta curricular clara, secuencial, pertinente y flexible que involucre procesos socioespaciales y de organización espacial, basados en el análisis, interpretación y criticidad. Es necesaria la construcción de ejes conceptuales que privilegien las relaciones estructurales del espacio con las dinámicas sociales en todas sus dimensiones; la consecución de materiales, como textos escolares o unidades didácticas, basados en los procesos de aprendizaje, la renovación disciplinary pedagógica, el uso de diferentes lenguajes, y una reflexión sobre el papel de la geografía en la escuela y su función social. Además, son indispensables todas aquellas iniciativas y propuestas que, desde la labor docente y los grupos académicos, propendan por el desarrollo de políticas y currículos pertinentes.

\section{Referencias}

Blanco, J. (2009). Espacio y territorio: elementos teórico-conceptuales implicados en el análisis geográfico. En Fernández, M. y Gurevich, R. (eds.), Geografía. Nuevos temas, nuevas preguntas, un temario para su enseñanza (pp. 37-64). Buenos Aires: Biblos.

Caballero, J. (2000). Didáctica de las ciencias sociales, geografía e historia para profesores de educación secundaria. Sevilla: MAD.

Castellar, S. (2014). Geografía Escolar, contextualizado a sala de aula. Curitiba: CRV.

Franco, M., Montañez, G., Rodríguez, E. y Torres, R. (2000). Propuesta para la enseñanza de la geografía en la educación básica. En Memorias xvı Congreso Colombiano de Geografía, Cali, Colombia.
Ministerio de Educación Nacional (MEN). (1996). Resolución 2343 del 5 de junio de 1996, por la cual se adopta un diseño de lineamientos generales de los procesos curriculares del servicio público educativo y se establecen los indicadores de logros curriculares para la educación formal. https://docplayer.es/8863816-Resolucion-2343-de-junio-5-de-1996.html

Ministerio de Educación Nacional (MEN). (2002). Lineamientos Curriculares Ciencias Sociales en Educación Básica. Bogotá: Imprenta Nacional de Colombia.

Ministerio de Educación Nacional (MEN). (2006). Estándares Básicos en Competencias en Ciencias Sociales y Ciencias Naturales. Bogotá: Imprenta Nacional de Colombia.

Ministerio de Educación Nacional (MEN). (2016). Derechos Básicos de Aprendizaje (DBA) ciencias sociales. Bogotá: Panamericana.

Ministerio de Educación Nacional (MEN). (2017). Resolución 18583 del 15 de septiembre de 2017, por la cual se ajustan las características específicas de calidad de los programas de Licenciatura para la obtención, renovación o modificación del registro calificado, y se deroga la Resolución 2041 del 2016. https://www.usbcali. edu.co/sites/default/files/resolucion_final_18583_de_2017deroga_2041.pdf

República de Colombia (1994). Ley 115 del 8 de febrero de 1994, por la cual se expide la ley general de educación. https://www.mineducacion.gov.co/1621/articles-85906_archivo_pdf.pdf

Rodríguez, E. (2000). Geografía conceptual, enseñanza y aprendizaje de la geografía en Educación Básica Primaria. Bogotá: Tercer Mundo.

Rodríguez, E. (2010). Geografía conceptual, enseñanza y aprendizaje de la geografía en Educación Básica Secundaria. Bogotá: Estudio Caos.

Rodríguez, E., Rodríguez, L. y Pérez, G. (2003). Algunas habilidades y procesos de pensamiento para comprender la dinámica espacial. Conjeturas, 7.

Rodríguez, L. (2007). Una geografía escolar (in)visible. Desarrollo de pensamiento espacial desde la construcción de conceptos geográficos. Bogotá: Universidad Distrital Francisco José de Caldas.

Souto, X. (1999). Didáctica de la geografía, problemas sociales y conocimiento del medio. Barcelona: Ediciones del Serbal.

Souto, X. (2017). Los métodos didácticos en la enseñanza del espacio geográfico. En Sebastiá, R. y Tonda, E. (eds.), Enseñanza y aprendizaje de la geografía para el siglo xxı (pp. 73-96). Alicante: Universitat D'Alacant. 